\title{
Research on Batch Evaluation of Real Estate Price Based on XGBoost
}

\author{
Likuang Zeng* \\ School of Economics and Management, Nanjinng University of Science and Technology, Nanjing, \\ China
}

*Corresponding author: 1094085907@qq.com

\begin{abstract}
Nowadays, there is an upsurge of research in the field of artificial intelligence around the world. As artificial intelligence slowly penetrates and is applied to all walks of life, the application of artificial intelligence to the field of real estate price batch evaluation has gradually become a reality. As the core area of artificial intelligence-machine learning, it has strong self-organization, selfadaptation and learning capabilities, and is very good at dealing with nonlinear mapping relations. It is a good choice to apply the ideas and methods of artificial intelligence to the field of real estate price batch evaluation. This paper uses Python web crawler technology to obtain Nanjing's secondhand housing transaction information data set from Shell.com, and cleans the second-hand housing transaction information data set to obtain a standardized data set. Then data processing is performed on the standardized data set, which mainly includes the quantitative processing of sub-type variables to obtain a standardized sample data set. Next, we build a real estate price batch evaluation model based on XGBoost, tune the main parameters of the regression model, and perform 5 -fold crossvalidation. Finally, we compare and analyze the XGboost regression model with the random forest regression model and the multiple regression model, and find that the XGBoost regression model has considerable advantages compared with the traditional multiple regression model, and is more suitable for building real estate price batch evaluation models; moreover, The real estate price batch evaluation model based on XGBoost is slightly better than the real estate price batch evaluation model based on random forest, and the regression prediction results are more robust.
\end{abstract}

Keywords: Batch evaluation of real estate prices; Machine learning; XGBoost.

\section{Introduction}

In recent years, as the expansion of urbanization has slowed and the market for new real estate has gradually become saturated, the real estate market has gradually shifted from an increase-based to a stock-based market, and the second-hand housing market has gradually become the main front of the real estate market. Taking Nanjing as an example, after a round of mandatory intervention measures in the real estate market (in 2016, first-tier cities strengthened purchase and loan restrictions, and many places have also introduced restrictions on purchases and loans), the number of second-hand housing transactions in Nanjing has increased from 151,524 sets per year in 2016, and then dropped to 72230 set s per year in 2018, a record low in 4 years. However, after a round of cooling in the second-hand housing market, the second-hand housing market in Nanjing began to pick up in 2019. The number of second-hand housing transactions reached 113,466 units per year in 2019, breaking the 100,000 mark again, and there has been a slow growth since then, with the number of transactions remaining at 10 Million plus.

However, as second-hand housing transactions become more active, the number of transactions continues to grow, and people's demand for information and data such as second-hand housing transaction prices is also growing. , Efficient and accurate real estate price batch appraisal system to meet market demand.

\section{Literature review}

The idea of batch appraisal of real estate prices can be traced back to the beginning of the 20th century, when foreign scholars proposed the use of multiple regression models for batch appraisal of 
real estate prices. In the 1970 s, the birth of computers and the revolution in information technology provided objective conditions for the realization of a computer-aided batch evaluation system. Theoretical and empirical studies on the batch evaluation of real estate prices have sprung up. One of the more typical is the characteristic price model. The characteristic price model is also called the Hedonic model. It was first proposed by Court in 1939. Later, it combined Lancaster's consumer preference theory (Lancaster, 1966) and Rosen's supply-demand equilibrium model (Rosen, 1974) to develop into a more mature mathematical model. Economic model. The model believes that the utility of real estate characteristics (such as building area, house orientation, location, etc.) will determine the price of real estate.

Ridker (1967), Robert A. Blettner (1969), Muth (1969) and others applied the Hedonic model to the field of housing price evaluation for the first time, proving that the utility of housing characteristics determines housing prices. Straszheim (1974) put forward the problem of residential market segmentation on this basis. He subdivided the urban residential market into several similar sub-markets, each of which corresponds to a different characteristic price function, and then constructed models for the sub-markets respectively, and had reduced the variance of the overall sample fit.

After the 1980s, the innovative development of real estate price batch evaluation theory was slow. Research on this aspect mainly focused on improving evaluation methods and technologies. At the end of the 1990s, Geographic Information System (GIS) was gradually applied to the field of batch evaluation of real estate prices. In 1997, Richard Borst, William McCluskey, William Ddeddis and others applied geographic information technology to batch evaluation. By combining the geographic information management system, a batch evaluation system was established, which greatly improved the efficiency and accuracy of the batch evaluation, and helped the later geography. The application of information management system in batch evaluation has laid the foundation.

\section{Empirical research}

\subsection{Sources of sample data}

As the leading enterprise in the national real estate intermediary market, Shell has a large-scale national database of second-hand housing transaction information. This article uses the Python crawler program to obtain second-hand housing transaction information data in Nanjing from September 2020 to September 2021 from Shell.com, and obtain two sample data sets. The two sample data sets were sample cleaned, and samples containing missing values and outliers were removed, and two standardized and effective sample data sets - Nanjing Housing Price Data Set were obtained. There were 10382 sample data and 17 data features. The data characteristics of the Nanjing housing price data set are as shown in Table 1:

Table 1. Data characteristics of the sample data set.

\begin{tabular}{|c|c|c|}
\hline type of data & Data label & Data content \\
\hline \multirow{3}{*}{ Continuous } & Deal price & Total transaction price of second-hand housing \\
\cline { 2 - 3 } & $\begin{array}{c}\text { Construction } \\
\text { area }\end{array}$ & Second-hand housing construction area \\
\cline { 2 - 3 } & Longitude & Longitude of second-hand housing \\
\cline { 2 - 3 } Discrete (numerical) & $\begin{array}{c}\text { Latitude } \\
\text { Construction } \\
\text { age }\end{array}$ & Latitude of second-hand housing \\
\hline Discrete (numerical) & $\begin{array}{c}\text { Number of } \\
\text { bedrooms }\end{array}$ & Number of bedrooms in second-hand houses \\
\hline Discrete (numerical) & $\begin{array}{c}\text { Number of } \\
\text { living rooms }\end{array}$ & Number of living rooms in second-hand housing \\
\hline
\end{tabular}




\begin{tabular}{|c|c|c|}
\hline Discrete(numerical) & $\begin{array}{c}\text { Number of } \\
\text { toilets }\end{array}$ & Number of bathrooms in second-hand houses \\
\hline Discrete (numerical) & $\begin{array}{l}\text { Number of } \\
\text { kitchens }\end{array}$ & Number of second-hand kitchens \\
\hline Discrete (numerical) & $\begin{array}{l}\text { Total number } \\
\text { of floors }\end{array}$ & $\begin{array}{l}\text { The total number of floors in the building where the } \\
\text { second-hand housing is located }\end{array}$ \\
\hline Discrete(classification) & $\begin{array}{l}\text { Floor of the } \\
\text { house }\end{array}$ & $\begin{array}{l}\text { The floor where the second-hand house is located, the } \\
\text { value is: low floor, middle floor, high floor }\end{array}$ \\
\hline Discrete(classification) & House structure & $\begin{array}{l}\text { Second-hand house type structure, the value is: split- } \\
\text { level, duplex, cline, and leveling }\end{array}$ \\
\hline Discrete(classification) & Building Type & $\begin{array}{c}\text { Second-hand housing construction type, the value is: } \\
\text { bungalow, tower, plate tower combination, slab } \\
\text { building }\end{array}$ \\
\hline Discrete(classification) & $\begin{array}{l}\text { Building } \\
\text { structure }\end{array}$ & $\begin{array}{l}\text { Second-hand building structure, the value is: steel- } \\
\text { concrete structure, steel structure, mixed structure, } \\
\text { frame structure, brick-concrete structure, brick-wood } \\
\text { structure }\end{array}$ \\
\hline Discrete(classification) & $\begin{array}{l}\text { House } \\
\text { orientation }\end{array}$ & $\begin{array}{l}\text { The orientation of the second-hand housing is: east, } \\
\text { south, west, north, northeast, southeast, southwest, } \\
\text { northwest, etc. }\end{array}$ \\
\hline Discrete(classification) & $\begin{array}{l}\text { Renovation } \\
\text { condition }\end{array}$ & $\begin{array}{l}\text { Second-hand house decoration situation, the value is: } \\
\text { rough, light-covered, hard-covered }\end{array}$ \\
\hline Discrete(classification) & $\begin{array}{l}\text { Equipped with } \\
\text { elevator }\end{array}$ & $\begin{array}{l}\text { Whether second-hand houses are equipped with } \\
\text { elevators, the value is: none, yes }\end{array}$ \\
\hline
\end{tabular}

\subsection{Sample data processing}

Before constructing a real estate price batch evaluation model, the input and output variables of the constructed model must be determined. The output variable represents the expected output target of the model, so this paper chooses the transaction price of second-hand housing as the output variable. The input variable is the factor that affects the real estate transaction price, that is, the data feature that deducts the transaction price is the input variable. According to Table 1, we can know that there are many discrete categorical variables in the input variables. These categorical variables have no specific values and are presented in text form. They cannot be directly imported into the machine learning model and need to be quantified. According to the characteristics of text-based categorical variables, we can quantify the variables by decomposing them into dummy sub-variables. Taking the data variable of the second-hand house 'floor of the house' as an example, let's explain the specific quantitative processing method. The original value of the input variable of the 'floor of the house' is: 'low floor', 'middle floor', and 'high floor'. We can decompose the text-based categorical variable of the floor where the house is located into three dummy sub-variables according to its original value content, namely, 'floor of the house_ low floor', 'floor of the house_ middle floor', and 'floor of the house_ high Floor', the value of the three dummy sub-variables is 0 or 1 . In this way, we can use these three dummy sub-variables to represent the value of the mother variable of 'the floor where the house is located', as shown in the following Table 2:

Table 2. The quantification method of the variable 'floor of the house'.

\begin{tabular}{|c|c|c|c|}
\hline $\begin{array}{c}\text { Floor of the house_ } \\
\text { low floor }\end{array}$ & $\begin{array}{c}\text { Floor of the house_ } \\
\text { middle floor }\end{array}$ & $\begin{array}{c}\text { Floor of the house_ } \\
\text { high floor }\end{array}$ & $\begin{array}{c}\text { Floor of the } \\
\text { house }\end{array}$ \\
\hline 1 & 0 & 0 & Low floor \\
\hline 0 & 1 & 0 & Middle floor \\
\hline 0 & 0 & 1 & High floor \\
\hline
\end{tabular}


We quantify other text-based categorical variables in the same way, and convert the original textbased categorical variables into numerical categorical variables. The value range of the new numerical categorical variable is $\{0,1\}$.

Table 3. Quantitative methods of text-based categorical variables.

\begin{tabular}{|c|c|}
\hline Text-based categorical variables & Numerical categorical variables \\
\hline \multirow{3}{*}{ Floor of the house } & Floor of the house_Low floor \\
\hline & Floor of the house_ Middle floor \\
\hline & Floor of the house_ High floor \\
\hline \multirow{4}{*}{ House structure } & Unit structure_Duplex \\
\hline & Unit structure_leveling \\
\hline & Unit structure_cline \\
\hline & Unit structure_Split level \\
\hline \multirow{4}{*}{ Building Type } & Building Type_Tower \\
\hline & Building Type_Bungalow \\
\hline & Building Type_Plate Tower Combination \\
\hline & Building Type_Slab Building \\
\hline \multirow{6}{*}{ building structure } & Building Structure_ Steel-Concrete Structure \\
\hline & Building Structure_Mixed Structure \\
\hline & Building structure_Brick-wood structure \\
\hline & Building structure_Brick-Concrete Structure \\
\hline & Building Structure_Steel Structure \\
\hline & Building Structure_Frame Structure \\
\hline \multirow{8}{*}{ House orientation } & House orientation_East \\
\hline & House orientation_Northeast \\
\hline & House orientation_Southeast \\
\hline & House orientation_North \\
\hline & House orientation_South \\
\hline & House orientation_West \\
\hline & House orientation_ Northwest \\
\hline & House orientation_Southwest \\
\hline \multirow{3}{*}{ Decoration situation } & Decoration situation_Rough \\
\hline & Decoration situation_Paperback \\
\hline & Decoration situation_Hardcover \\
\hline \multirow{2}{*}{ Equipped with elevator } & Equipped with elevator_No \\
\hline & Equipped with elevator_Yes \\
\hline
\end{tabular}

\subsection{Batch evaluation of real estate prices based on XGBoost}

XGBoost is an integrated learning algorithm based on the decision tree algorithm and improved on the gradient boosting tree algorithm (GBDT). This algorithm was proposed by Dr Chen Tianqi of the University of Washington in February 2014. It has high training performance and its application field is continuously expanding, gradually expanding from the field of industrial production to the field of economic analysis. The XGBoost algorithm is an improvement on the original gradient boosting tree, and a regularization term is added to the loss function to impose a penalty on the complexity of the model to improve the generalization ability of the model and prevent overfitting; at the same time, the XGBoost algorithm is parallelized and distributed. The formula ability has also greatly improved its training efficiency. Compared with the gradient boosting tree, it has higher adaptability and stronger robustness, and it also improves the accuracy of data prediction and training speed. Based on the basic theory of XGBoost model, this paper builds XGBoost regression model in Python environment. The specific operation is: import the XGBRegressor function from the Python 
xgboost. Sklearn library, set and optimize the corresponding parameters of the XGBRegressor function, and then establish a real estate price batch evaluation model based on the Nanjing housing price data set.

The following are the main parameter settings of the XGBoost regression model:

Table 4. Main parameter settings of XGBoost regression model.

\begin{tabular}{|c|c|c|}
\hline parameter name & Parameter Description & $\begin{array}{c}\text { Parameter } \\
\text { setting }\end{array}$ \\
\hline booster & The type of weak learner, the default value is 'gbtree' & gbtree \\
\hline n_estimators & The number of base learner gradient boosting trees & 1000 \\
\hline max_depth & $\begin{array}{c}\text { The maximum depth of the base learner gradient boosting tree, } \\
\text { the default value is 6 }\end{array}$ & 7 \\
\hline gama & $\begin{array}{c}\text { The penalty term coefficient specifies the minimum loss } \\
\text { function decline value required for leaf node splitting. Default } \\
\text { is 0 }\end{array}$ & 0.4 \\
\hline min_child_weight & $\begin{array}{c}\text { The smallest sample weight sum in the leaf node. If the sum of } \\
\text { the sample weights of a leaf node is less than min_child_ } \\
\text { weight, the splitting process ends. In the regression model, the } \\
\text { minimum number of samples required for each model is } \\
\text { expressed. Default is 1 }\end{array}$ & 2 \\
\hline learning_rate & The learning rate of the model, the default value is 0.3 & 0.05 \\
\hline
\end{tabular}

We import the sample data set into the parameterized XGBoost regression model, and after fivefold cross-validation, the following regression results are obtained:

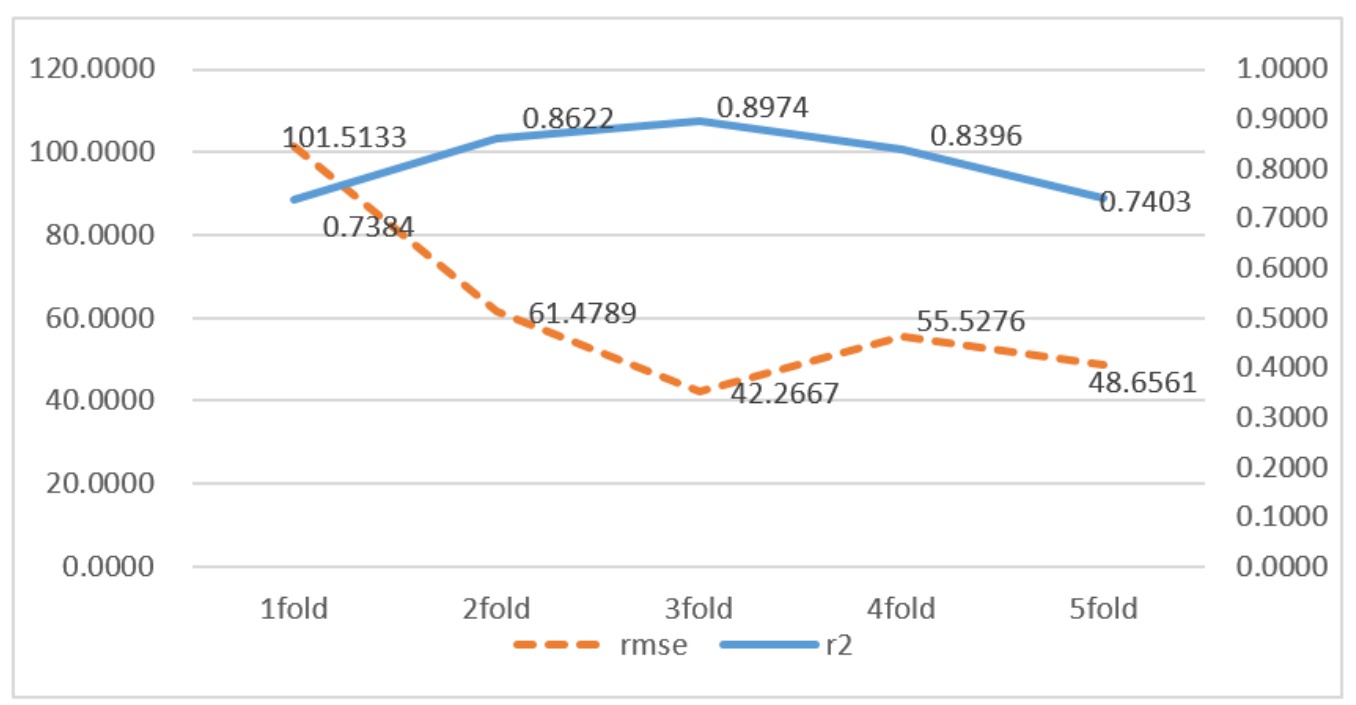

Figure 1. XGBoost regression results.

Note: (1) fold represents the fold number of cross-validation of sample data. 5-fold crossvalidation means that the sample data is divided into 5 groups and undergoes 5 cross-validation. The specific algorithm flow of 5-fold cross-validation is: first select the first group (ie 1 fold) as the test set, and the remaining 4 groups as the training set; then select the second group (2fold) as the test set, and the remaining 4 groups as the training set, 3 fold, 4 fold, 5fold and so on. (2) According to the definition of 5-fold cross-validation, the result of 5-fold cross-validation is the regression result of the sample data test set. After 5 times of cross-validation, 5 test sets can be obtained. Correspondingly, the regression evaluation index ( 2 , rmse) is also 5 values will be returned, and we can take the average of the 5 values of the regression evaluation index as an index to measure the predictive performance of the regression model. 
From Figure 1, we can know that the test set goodness of fit $\mathrm{r} 2$ of the 5-fold cross-validation first increases and then decreases with the test set fold number. The specific performance is: when the test set fold number reaches 3 fold, the test set fits The goodness reached the maximum value of 0.8974 , and then as the fold number of the test set continued to decrease, and finally decreased to 0.7403; the root mean square error rmse of the test set for 5-fold cross-validation first decreased and then increased with the fold number of the test set Big and then decrease, the specific performance is: the rmse of the test set first decreases to the minimum value of 42.2667 with the test set fold number, at this time the test set fold number is 3 fold, when the test set fold number is 4 fold, rmse rises to 55.5276 , And finally reduced to 48.6561. From the change of the test set $\mathrm{r} 2$ and rmse with the test set fold number, we can know that the distribution of the sample data is not balanced. This unbalanced data distribution feature has a certain impact on the model's regression prediction ability. Therefore, the regression evaluation index of a single test set cannot measure the predictive ability of the XGBoost regression model. For this reason, we take the average of the regression results of the 5 test sets and use it as an evaluation index to measure the XGBoost regression model. According to Figure 3.1, we can get the average of the regression results of 5-fold cross-validation, where the average of $\mathrm{r} 2$ is 0.8156 and the average of rmse is 61.8885 .

At the same time, we put the true and predicted values of the 5 test sets in the 5 -fold crossvalidation into one graph, and then we can get the following scatter plot:

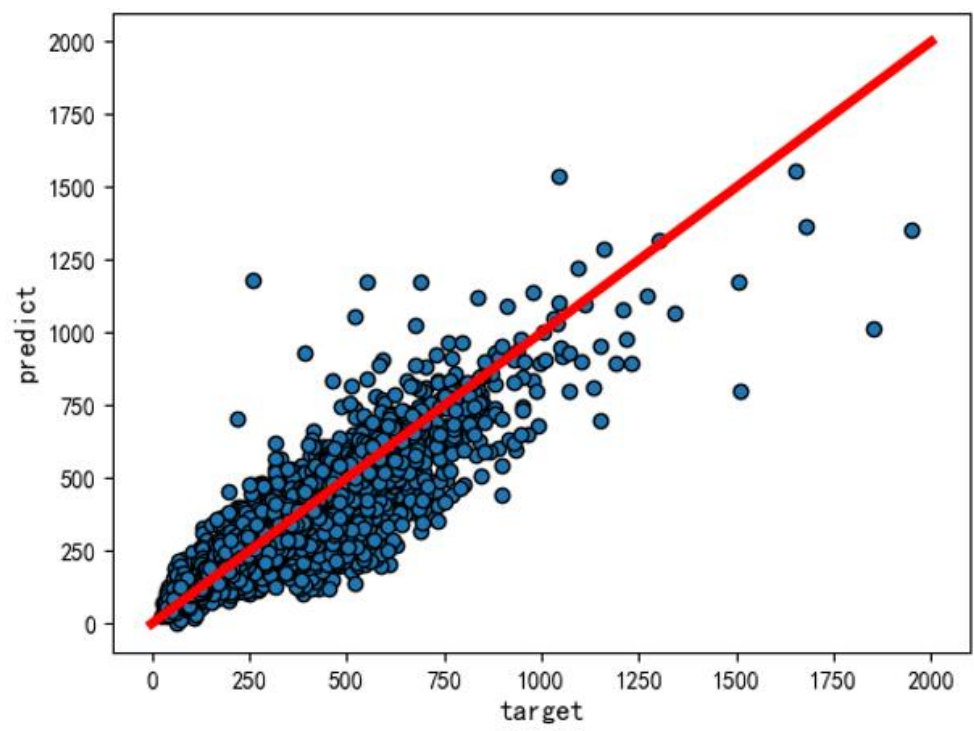

Figure 2. Target \& predict.

According to Figure 2, we can clearly find that the absolute majority (true value, predicted value) of the scattered points are concentrated near the line $y=x$, and a few scattered points are far away from $\mathrm{y}=\mathrm{x}$, which also shows that based on the Nanjing housing price data set The prediction performance of the XGBoost regression model is quite good.

A detailed analysis of the XGBoost-based real estate price batch appraisal model was made in the previous section. Next, we will compare and analyze the real estate price batch appraisal model based on multiple regression, the real estate price batch appraisal model based on random forest, and the real estate price batch appraisal model based on XGBoost.

Table 5. Comparison of the results of the three regression models.

\begin{tabular}{|c|c|c|}
\hline & r2 & rmse \\
\hline Multiple Regression & 0.507 & 101.7251 \\
\hline Random forest & 0.7838 & 65.8176 \\
\hline XGBoost & 0.8156 & 61.8885 \\
\hline
\end{tabular}


According to Table 5, the prediction performance of the XGBoost regression model is much higher than that of the multiple regression model. This is not only reflected in the XGBoost regression model's $\mathrm{r} 2$ is about $60 \%$ higher than the multiple regression model, but also reflected in the mean square of the XGBoost regression model is $40 \%$ lower than the multiple regression model, which shows that the application of XGBoost machine learning algorithm in the batch evaluation of real estate prices is feasible and has considerable advantages compared to the multiple regression model. From Table 5, we can know that the goodness of fit of the XGBoost regression model is higher than that of the Random Forest regression model, and the root mean square error of the XGBoost regression model is also lower than that of the Random Forest regression model. From this we can know that the regression prediction effect of the real estate price batch evaluation model based on XGBoost is slightly better than the Random Forest regression model.

\section{Summary}

At present, local urbanization is slowing down, the new real estate market is gradually becoming saturated, and the second-hand housing market has gradually become the main front of the local urban real estate market. However, with the gradual expansion of the second-hand housing market, its transaction scale is also growing rapidly, and people's demand for information and data such as real estate transaction prices is also increasing. Local cities urgently need an intelligent and automated real estate price batch evaluation system, to timely, efficiently and accurately reflect information and data such as real estate transaction prices. However, local cities also need to pay attention to some issues when building a similar intelligent and automated real estate price batch evaluation system such as a second-hand housing transaction data sharing platform: (1) A complete housing transaction information database needs to be established, covering the characteristics and location of housing construction Feature data such as features provide sample data for the iterative update of the batch evaluation system. In addition, it is also necessary to establish a set of standard and standard index systems to measure or describe the location characteristics of houses (such as transportation convenience, living facilities, education facilities, commercial service facilities, community properties, etc.), as well as building features, location features, etc. The input indicators construct a set of quantitative standards, which lay the foundation for the training and testing of regression prediction models. (2) When establishing a real estate price batch evaluation system, you can consider using machine learning algorithms such as XGBoost and random forest as the core algorithm framework of the batch evaluation system to build a real estate price batch evaluation model based on machine learning algorithms. This can not only improve the evaluation efficiency, but also can improve the accuracy of the assessment.

\section{References}

[1] Carbone Robert, Longini Richard. A Feedback Model for Automated Real Estate Assessment [J]. Management Science, 1977, 24(3): 241-248

[2] William McCluskey, William Deddis, Adam Mannis, Dillon Mcburney, Richard borst. Internactive application of computer assisted mass appraisal and geographic informationsystems [J]. Journal of Property Valuation and Investment, 1997, 15(5): 448-465

[3] Arvydas Bagdonavicius Steponas Deveikis. Individual and Mass Valuation-Present and Future Practices [J]. Valuation and Real Estate Management, 2005(04): 16-21

[4] John D Benjamin, Randall S. Guttery, C.F.Sirmans. Mass appraisal: an introduction to multiple regression analysis for real estate valuation [J]. Journal of Real Estate Practice and Education. 2004, 7(1):65-77

[5] Vilius Kontrimas, Antanas Verikas. The mass appraisal of the real estate by computational intelligence [J]. Apllied Soft Computing, 2011, 11:443-448

[6] Al-Akhras, Mousa. An evolutionary-optimised artificial neural network approach for automatic appraisal of Jordanian lands and real properties [J]. World Scientific and Engineering Academy and Society, 2010, 6, 203-208 
[7] Evgeny A. Antipov, Elena B. Pokryshevskaya. Mass appraisal of residential apartments: An application of Random forest for valuation and a CART-based approach for model diagnostics [J]. Expert Systems with Applications. 2012(39): 1772-1778

[8] Peterson S, Flanagan AB. Neural network hedonic pricing models in mass real estate appraisal. Journal of Real Estate Research, 2009, 31(2): 147-164.

[9] Riddker, Ronald G, Henning, et al. The Determinants of Residents Property Values with Special Reference to Air Pollution. Review of Economics \& Statistics, 1967: 246-247

[10] Chica-Olmo J. Prediction of housing location price by a multivariate spatial method: cokriging. Journal of Real Estate Research, 2007, 29(1): 91-114

[11] Tay DPH, Ho DKH. Artificial intelligence and the mass appraisal of residential apartments. Journal of Property Valuation and Investments. 1992, 10(2): 525-540

[12] Kathman RM. Neural networks for the mass appraisal of real estate. Computers, environment and urban systems, 1993, 17(4): 373-384

[13]Hakankusan, OsmanAythekin. The use of fuzzy logic in predicting house selling price. Expert Systems with application, 2010, 37(3): 1808-1813 